\title{
Preparation of Fluorine-Free Superhydrophobic Paper with Dual-Response of Temperature and $\mathrm{pH}$
}

\author{
Bin Du ${ }^{1,2}$, Daodao Xue ${ }^{1}$, Rubai Luo ${ }^{1,2, *}$, Huailin Li ${ }^{1,2}$, Kenan Yang ${ }^{3}$ and Shisheng Zhou ${ }^{1,2}$ \\ 1 Faculty of Printing, Packaging Engineering and Digital Media Technology, Xi'an University of Technology, \\ Xi'an 710048, China; dubin@xaut.edu.cn (B.D.); 2190821080@stu.xaut.edu.cn (D.X.); \\ lihuailin@xaut.edu.cn (H.L.); zhoushisheng@xaut.edu.cn (S.Z.) \\ 2 Shaanxi Provincial Key Laboratory of Printing and Packaging Engineering, Xi'an University of Technology, \\ Xi'an 710048, China \\ 3 Faculty of Mechanical and Precision Instrument Engineering, Xi'an University of Technology, Xi'an 710048, \\ China; 2170820027@stu.xaut.edu.cn \\ * Correspondence: luorubai@xaut.edu.cn; Tel.: +86-132-2771-4944
}

Received: 27 September 2020; Accepted: 27 November 2020; Published: 29 November 2020

\begin{abstract}
Although various superhydrophobic materials have been manufactured and effectively used for oil-water separation, it is still highly desirable to explore materials which are eco-friendly, low-cost, and multifunctional. In this paper, a stable copolymer solution was prepared from the fluorine-free superhydrophobic copolymer with dual-responsiveness of temperature and $\mathrm{pH}$. The functional superhydrophobic paper was prepared by immersing paper in copolymer solution by the dip-coating method. The surface element and structure analysis of the prepared superhydrophobic paper shows that the dual-responsive copolymer adheres successfully to the surface of the paper without destroying the fiber structure of the paper. At $\mathrm{pH} \geq 7$ and $T>25^{\circ} \mathrm{C}$, the paper has a good superhydrophobic performance, while under the conditions of $\mathrm{pH}<7$ and $T<25^{\circ} \mathrm{C}$, the paper comes into a hydrophilic state. Therefore, the dual-responsive superhydrophobic paper is more likely to adapt to the complicated oil-water separation environment than the single-response.
\end{abstract}

Keywords: superhydrophobic; dual-response; oil-water separation; contact angle

\section{Introduction}

The wettability of a solid surface depends on its surface chemical composition and microscopic geometry. This is an important performance index for many processes and applications and has aroused extensive research interest in researchers in recent decades [1-3]. In particular, a surface with a static water contact angle larger than $150^{\circ}$ and a sliding angle lower than $10^{\circ}$ is called a superhydrophobic surface [4,5]. Due to its excellent characteristics of self-cleaning [6], oil-water separation [7], water-proof [8], anti-fouling [9], anti-icing [10] and anti-fogging [11], it has been favored by material workers for many years, attracting a large number of researchers to study the performance of superhydrophobic materials. At present, it has been found that superhydrophobic surfaces can be fabricated on silicon wafers [12], glass [13], metals [14], plastics [15], textiles [16], wood [17,18], and paper [19]. In fact, the premise of becoming a superhydrophobic material based on previous research is that a superhydrophobic material must have both a micro-nano-composite structure and low-surface energy chemicals [20,21].

Paper is a common and indispensable material in our lives. It has a wide range of uses. Due to its lightweight, portability, easy transmission and communication, low price, high flexibility, biodegradability and renewable properties, it has been favored by researchers. Research is concerned especially with its adaption to the complex external environment and its ability to meet the special needs 
of merchants for paper. The demand for modified paper is also increasing in the market, including that for superhydrophobic paper, which can be used for moisture-proof packaging [22], paper-based sensors [23], oil-water separation and self-cleaning materials [24]. There are many preparation methods for superhydrophobic paper, mainly including the dipping method, the chemical etching method, the electrophoretic deposition method, the electrospinning method, the chemical vapor deposition method, the plasma etching method, and the spraying method, etc. [25]. However, in these methods, some of the reagents used in the preparation process will be harmful to the environment, and some preparation processes and required equipment are complicated, so it is very important to choose the appropriate reagents and preparation methods. Besides, it is necessary to broaden the application range of superhydrophobic paper and make it have a better environmental adaptability. In recent years, some sensitive polymers with environmental irritation have been developed by researchers to respond to various stimuli in the external environment, including temperature, $\mathrm{pH}$, light, ultrasound, electron transfer (redox change), etc. [26-37], in which temperature and $\mathrm{pH}$ are the most commonly used stimuli. Combining any two of the above stimuli can also prepare intelligent interface materials at the air/solid interface. Compared with single stimuli, intelligent materials with double stimuli have better environmental adaptability, and people are increasingly studying dual-responsive intelligent polymers $[38,39]$. Moreover, the massive discharge of industrial oily wastewater not only poses huge challenges to the ecological environment but also seriously harms human health [40]. Thus, for the sake of human health and the harmony of the ecosystem, it is urgent to adopt environmentally friendly and effective methods to treat the oil pollution in industrial wastewater. The ecologically harmless superhydrophobic paper with dual- responsiveness offers a new way to solve the problem. Therefore, it is necessary to conduct in-depth research on dual-responsive polymers for expanding the application range of superhydrophobic paper so that it can better play its advantages.

In this study, N-isopropylacrylamide (NIPAm) and 2-(dimethylamino) ethyl methacrylate (DMAEMA) were selected as fluorine-free monomers for temperature and $\mathrm{pH}$-response, respectively. The superhydrophobic copolymer with a dual-response of temperature and $\mathrm{pH}$ was synthesized by a free radical solution polymerization method, and the pre-treated paper was immersed in the copolymer solution by a simple and easy-to-operate dip-coating to prepare the functional superhydrophobic paper. This work introduces a simple and fluorine-free superhydrophobic paper preparation method. The paper obtained by this method can make corresponding hydrophilic/hydrophobic reactions to the changes of temperature and $\mathrm{pH}$.

\section{Experimental Details}

\subsection{Materials}

Butyl methacrylate (BMA), Hydroxyethyl methacrylate (HEMA), 2-(Dimethylamino) ethyl methacrylate (DMAEMA) and N-isopropylacrylamide (NIPAm) were provided by Shanghai Aichun Biological Technology Co., Ltd. (Shanghai, China). Azobisisobutyronitrile (AIBN) was purchased from Tianjin Damao Chemical Reagent Factory (Tianjin, China). Absolute ethanol and n-hexane were provided by Tianjin Fuyu Fine Chemical Co., Ltd. (Tianjin, China). Acetone, anhydrous toluene, hydrochloric acid, and sodium hydroxide were provided by Tianjin Komiou Chemical Reagent Co., Ltd. (Tianjin, China). All reagents were analytical grade and used without other treatment.

\subsection{Methods}

\subsubsection{Synthesis of Superhydrophobic Copolymer with Dual-Responsiveness of Temperature and $\mathrm{pH}$}

The synthesis of superhydrophobic copolymer with dual-responsiveness of temperature and $\mathrm{pH}$ was carried out in the absence of water and oxygen by free radical polymerization, the synthetic route as shown in Figure 1. Taking the BMA (5.68 g, $0.04 \mathrm{~mol})$, HEMA (0.52 g, $0.004 \mathrm{~mol})$, DMAEMA (6.28 g, $0.04 \mathrm{~mol})$, NIPAm $(4.52 \mathrm{~g}, 0.04 \mathrm{~mol})$ and AIBN $(0.16 \mathrm{~g}, 0.001 \mathrm{~mol})$ respectively dissolved in $50 \mathrm{~g}$ of 
anhydrous toluene, after mixing, the prepared solution was poured into a three-necked round bottom flask. Then the flask was filled with nitrogen for $40 \mathrm{~min}$ to perform an oxygen removal operation, providing an oxygen-free environment for the polymerization reaction. After the deaeration with nitrogen was completed, the flask was sealed and placed in a thermostatic water bath at $65^{\circ} \mathrm{C}$ for $4 \mathrm{~h}$ to create a polymerization reaction. When the reaction was completed, the solution was left to cool to room temperature. After most of the solvent was slowly evaporated, it was dissolved in acetone, and then added to n-hexane under magnetic stirring to precipitate. The obtained product was dried in vacuum at $50{ }^{\circ} \mathrm{C}$ for $12 \mathrm{~h}$, after grinding into powder, a superhydrophobic copolymer with dual-responsiveness of temperature and $\mathrm{pH}$ could be obtained.
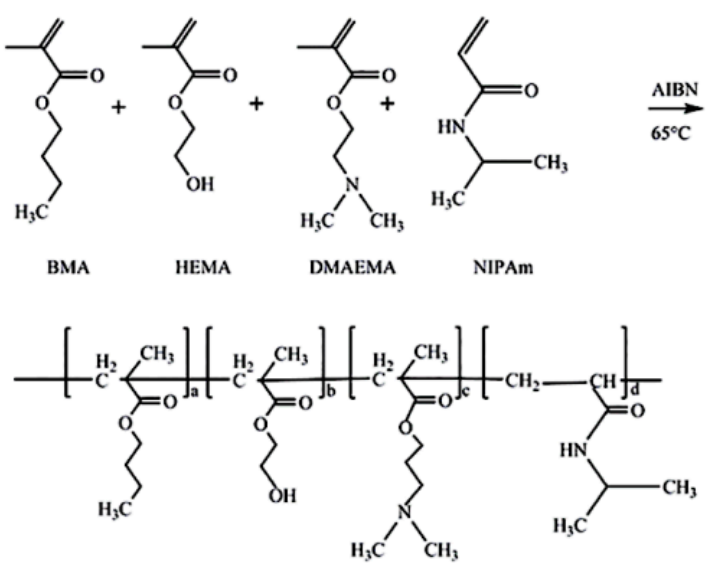

PBMA-O-PHEMA-O-PDMAEMA-CO.PNIPAm

Figure 1. The synthesis route of PBMA-co-PHEMA-co-PDMAEMA-co-PNIPAm.

\subsubsection{Fabrication of Temperature and $\mathrm{pH}$ Dual-Responsive Superhydrophobic Paper}

First, $1 \mathrm{~g}$ of the temperature and $\mathrm{pH}$ dual-responsive copolymer prepared above was added to $20 \mathrm{~g}$ of anhydrous toluene, and then dispersed and reacted under ultrasonic conditions for $30 \mathrm{~min}$ to form a uniform and stable copolymer solution. Then, the spare paper (German duni wood pulp paper, size 40 $\times 40 \mathrm{~cm}^{2}$, gram weight about $60 \mathrm{~g}$ ) was cut to a size of $3 \times 3 \mathrm{~cm}^{2}$. Acetone, anhydrous ethanol and deionized water were used to wash the paper under ultrasonic at $25{ }^{\circ} \mathrm{C}$ at room temperature and dried at $60^{\circ} \mathrm{C}$. Then the clean and the dry paper was soaked in the prepared copolymer solution for $30 \mathrm{~min}$ to form a superhydrophobic coating on its surface. The coated paper was put into a vacuum drying oven, and dried for about $2 \mathrm{~h}$ at $80^{\circ} \mathrm{C}$ to obtain a superhydrophobic paper with dual-responsiveness of temperature and $\mathrm{pH}$. The paper preparation process and the oil-water separation effect in response to temperature and $\mathrm{pH}$ are shown in Figure 2. The lower critical solution temperature (LCST) of the paper in the picture will be determined below.

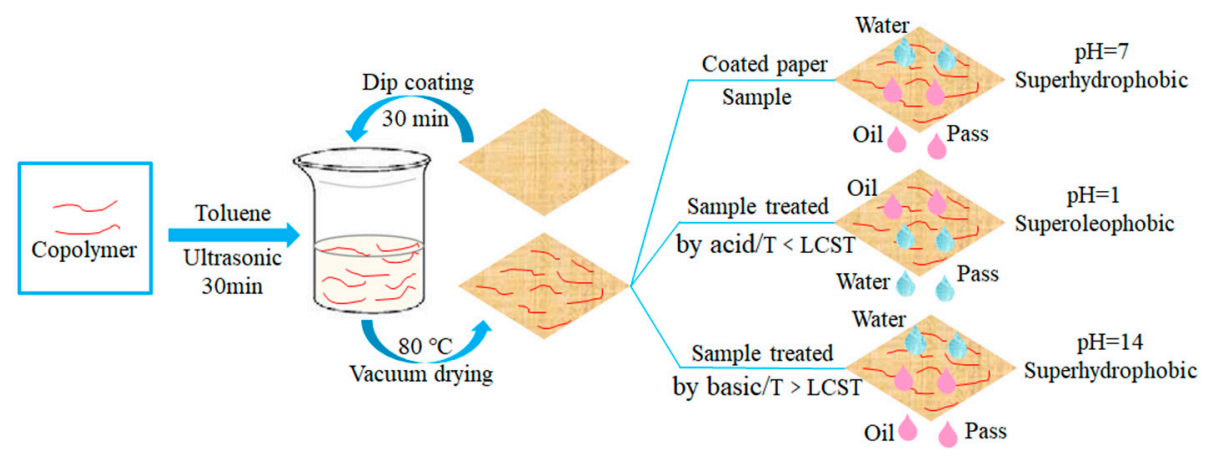

Figure 2. Synthesis procedure of temperature and $\mathrm{pH}$ dual-response superhydrophobic paper and its applications in oil/water separation under different temperature and $\mathrm{pH}$ treatments. 


\subsubsection{Determination of the Critical Temperature}

The LCST the of temperature and $\mathrm{pH}$ dual-responsive copolymer was measured by a differential scanning calorimeter (DSC) (TA Instruments, New Castle, DE, USA). The temperature at which phase transition occurs, LCST, was measured as peak temperature causing exothermic heat change.

To determine the LCST of the prepared dual-responsive superhydrophobic paper, five identical oil-water mixtures ( $20 \mathrm{~mL}$ oil-red O stained $\mathrm{n}$-hexane and $20 \mathrm{~mL}$ deionized water) were prepared in the beaker. By adjusting the temperature of the water bath, the temperature of the oil-water mixture was adjusted to $10,20,35,50$, and $65^{\circ} \mathrm{C}$, respectively. The specific operation process is shown in Figure 3 . The sample bottles commonly used in the experiment were processed. First, a nearly round hole was dug on the surface of the bottle cap and the bottle cap was unscrewed. The mouth of the sample bottle was sealed with modified paper coated with a copolymer. Then the lid was closed and the sample bottle was dipped into beakers at different water bath temperatures, making the water or oil in the oil-water mixture in the beaker able to enter the sample bottle through the paper under the cap of the round hole. Thus the separation was complete and the oil and water was collected. The results of oil-water separation were observed, and the temperature gradient was gradually reduced by this method until LCST was obtained.

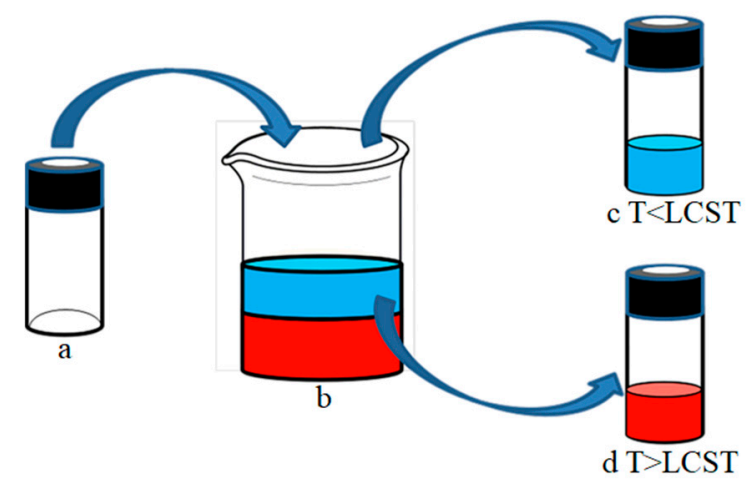

Figure 3. Simulation diagram of the oil-water separation process. (a) Perforated sample bottles sealed with copolymer-coated paper; (b) oil-water mixture; (c) deionized water was collected in the sample bottle when $T<$ LCST (d) red n-hexane was collected in the sample bottle when $T>$ LCST.

\subsection{Characterization}

The surface morphologies of the original paper and the coated paper were examined by a field emission scanning electron microscope (SEM) (SU-8010, Hitachi, Tokyo, Japan). The Fourier Transform infrared Spectroscopy (FTIR) was recorded using an infrared spectrometer (8400S, Shimadzu, Kyoto, Japan) at room temperature. The NIPAm monomer and the synthesized copolymer (PBMA-co-PHEMA-co-PDMAEMA-co-PNIPAm) were prepared by a $\mathrm{KBr}$ tableting method, and then the sample was measured using FTIR to observe the functional group of the product to verify the success of the polymerization. The structure of the copolymer dissolved in $\mathrm{C}_{3} \mathrm{D}_{6} \mathrm{O}$ was also analyzed by ${ }^{1} \mathrm{H}$ nuclear magnetic resonance $\left({ }^{1} \mathrm{H}\right.$ NMR) (AVANCE III HD $600 \mathrm{MHz}$, Bruker, Billerica, MA, USA) and all chemical shifts were recorded in ppm. The surface chemical compositions of the surface of the raw paper and copolymer-coated paper were carefully detected by an Axis Ultpa (Kratos Analytical, Manchester, UK) X-ray photoelectron spectroscopy (XPS). Al/K $\alpha(1486.71 \mathrm{eV})$ was used as a ray, and it was operated at a current of $10 \mathrm{~mA}$ and a voltage of $10 \mathrm{kV}$. The LCST of copolymer was measured under a nitrogen atmo-sphere by using a differential scanning calorimeter (DSC Q20, TA Instruments, New Castle, DE, USA). The heating rate was $1{ }^{\circ} \mathrm{C} \mathrm{min}-1$. The temperature at which phase transition occurs, LCST, was measured as a peak temperature causing exothermic heat change. Thermogravimetric analysis (TGA) was performed using a TA TGA-HP50 (TA Instruments, New Castle, DE, USA) thermogravimetric analyzer under a nitrogen atmosphere, and the temperature rose $\left(10^{\circ} \mathrm{C} \cdot \mathrm{min}^{-1}\right)$ from room temperature to $700^{\circ} \mathrm{C}$. The original paper and the copolymer-coated paper were subjected 
to thermogravimetric analysis. The wetting angle was measured by an SDC-200 (Dongguan Shengding Precision Instrument Co., Ltd., Dongguan, China) water contact angle measurement instrument. The test objects were untreated raw paper, copolymer-coated paper, and modified paper treated with different temperatures and $\mathrm{pHs}$. The paper soaked in the responsive solution was processed at different temperatures, and the water contact angle was measured. The paper soaked in $\mathrm{pH}$-responsive solution was immersed in a hydrochloric acidic solution $(\mathrm{pH}=1)$, a neutral solution (hydrochloric acid and a sodium hydroxide are not added, $\mathrm{pH}=7)$, and sodium hydroxide solution $(\mathrm{pH}=14)$ for $3 \mathrm{~min}$, and was then taken out in an oven at $60^{\circ} \mathrm{C}$ under vacuum drying, and the water contact angle was measured.

\section{Results and Discussion}

\subsection{Analysis of Paper Surface Morphology}

To study the effect of copolymer PBMA-co-PHEMA-co-PDMAEMA-co-PNIPAm on the surface morphology of paper, the surface morphology of raw paper, and modified paper soaked by copolymer solution were observed and analyzed by using SEM images with different magnifications. Figure $4 a-c$ are the original paper samples. On the whole, it can be seen that the surface of the paper fiber before the modification has only a small amount of impurities attached, and it is almost smooth and flat, and there is no obvious rough structure similar to protrusions. Figure $4 \mathrm{~d}-\mathrm{f}$ are the modified paper samples. It can be clearly seen that the surface of the paper fiber after coating with the copolymer has raised structures and wrinkles, which are no longer flat and smooth, and has become very rough. Compared with the relatively smooth surface of the original paper, it can be preliminarily judged that the modified paper is obviously rough because it is coated with a temperature and $\mathrm{pH}$ dual-responsive copolymer. Besides, by observing Figure $4 \mathrm{~d}-\mathrm{f}$, it is found that the surface of the modified paper fiber becomes rough only. Its structure and outline have not changed significantly, and the inherent distance between the fibers are still maintained. It ensures that the good mechanical and air permeability properties of the paper will not be changed. This shows that the surface coating of the original paper will not damage the fiber structure of the paper and affect some of its properties. Conversely, the paper can be coated with functional substances on the surface, which make it rougher and enhances the hydrophobic properties of the paper.

In addition, the surface morphology of the original paper and modified paper treated with acid and alkali were also observed, as shown in Figures 5 and 6. The surface of the original paper is still relatively smooth. The modified paper also retains its rough structure. This shows that acid and alkali treatment basically does not affect the surface morphology of paper fibers.

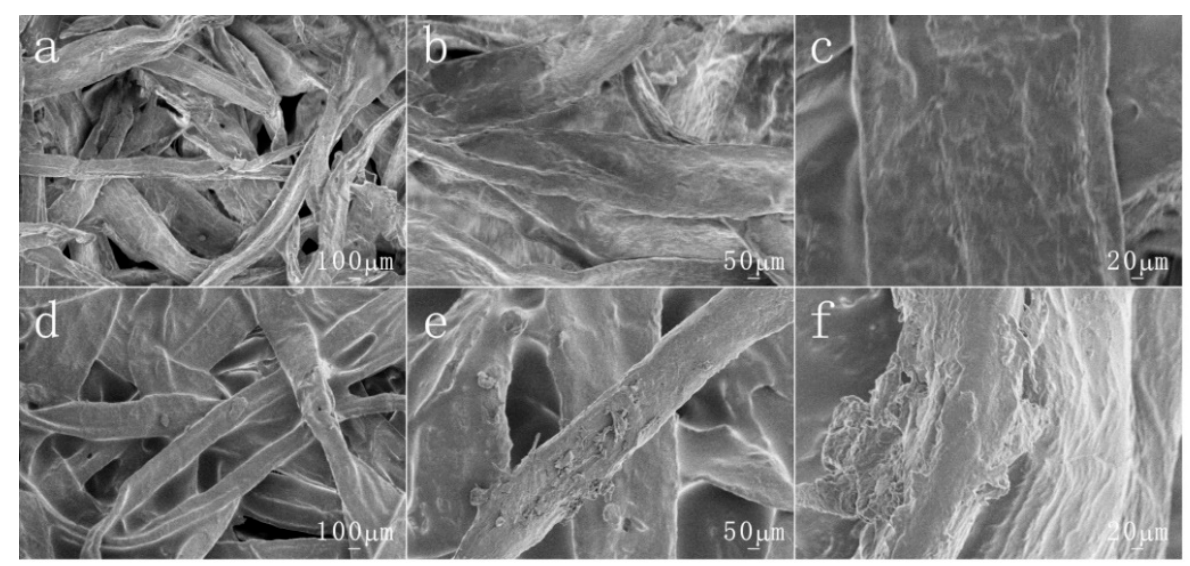

Figure 4. The different magnifications of SEM images of $(\mathbf{a}-\mathbf{c})$ pristine paperand $(\mathbf{d}-\mathbf{f})$ the copolymer coated paper. 


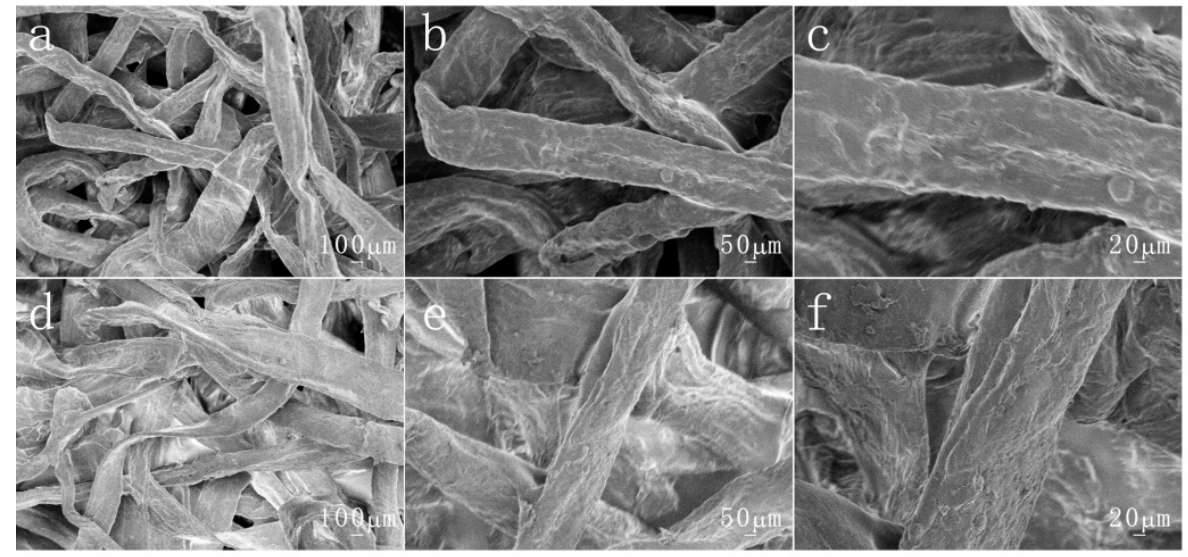

Figure 5. The different magnifications of SEM images by hydrochloric acid-treated $(\mathrm{pH}=1)(\mathbf{a}-\mathbf{c})$ pristine paper and $(\mathbf{d}-\mathbf{f})$ the copolymer-coated paper.

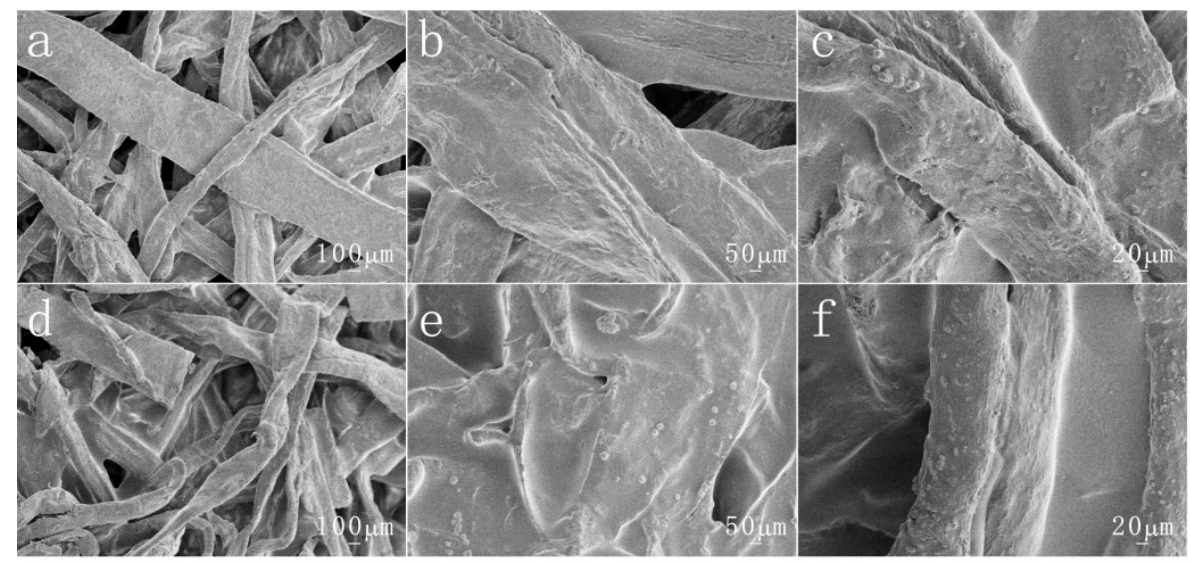

Figure 6. The different magnifications of SEM images by sodium hydroxide-treated $(\mathrm{pH}=14)(\mathbf{a}-\mathbf{c})$ pristine paper and $(\mathbf{d}-\mathbf{f})$ the copolymer-coated paper.

\subsection{Analysis of the Copolymer Structure}

As shown in Figure 7, the molecular structure of the temperature and the $\mathrm{pH}$ dual-responsive copolymer were characterized by ${ }^{1} \mathrm{H}$ NMR. The $-\mathrm{CH}_{2}$ peak of PBMA was observed at $1.4 \mathrm{ppm}$. The peaks observed at 2.23 and $2.3 \mathrm{ppm}$ correspond to $-\mathrm{N}-\left(\mathrm{CH}_{3}\right)_{2}$ and $-\mathrm{N}-\mathrm{CH}_{2}$ of PDMAEMA. The groups obtained at $4.07 \mathrm{ppm}$ contributed to $-\mathrm{CH}_{2} \mathrm{OH}$ and $-\mathrm{CH}_{2}$ linked to an ester group of PDMAEMA. The peak observed at $3.68 \mathrm{ppm}$ was due to $-\mathrm{CH}-\left(\mathrm{CH}_{3}\right)_{2}$ of PNIPAm.

FTIR was used to test and characterize the monomers used in the copolymerization reaction and the finally synthesized copolymer with dual-responsiveness. The chemical structure of the product was observed to further demonstrate the success of the polymerization. Figure 8 show the infrared spectra of the monomers BMA, HEMA, DMAEMA, NIPAm and the synthesized dual-responsive copolymer used for polymerization, respectively. In Figure 8 (green curve) the copolymer spectrum, the absorption peak at $2958 \mathrm{~cm}^{-1}$ corresponded to the stretching vibration of $-\mathrm{CH}$ in the monomers of BMA, HEMA, NIPAm and DMAEMA. The peak near $3533 \mathrm{~cm}^{-1}$ was ascribed to the stretching vibration of -OH in the HEMA monomer. The characteristic absorption bands at 1166 and $1720 \mathrm{~cm}^{-1}$ corresponded to the $\mathrm{C}-\mathrm{N}$ and $\mathrm{C}=\mathrm{O}$ tensile groups in DMAEMA, respectively. The stretching and bending vibration peaks of N-H were clearly observed at 3298 and $1546 \mathrm{~cm}^{-1}$, which were the typical absorption bands of NIPAm. The peak near $1668 \mathrm{~cm}^{-1}$ was attributed to the $\mathrm{C}=\mathrm{O}$ stretching vibration of NIPAm [41]. Through analysis, it was found that the main characteristic peaks of these monomers existed at the same wavelength in the spectra of the copolymer with dual-responsiveness. This phenomenon further indicated that the copolymer has been successfully synthesized from the microscopic point of view. 


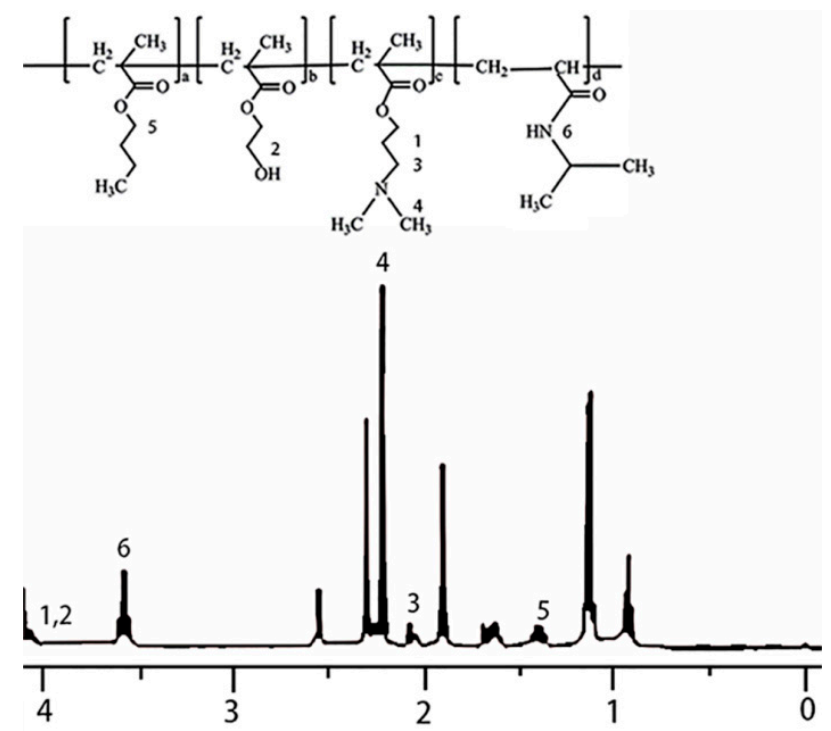

Figure 7. ${ }^{1} \mathrm{H}$ NMR spectrum of the temperature and the $\mathrm{pH}$ dual-responsive copolymer.

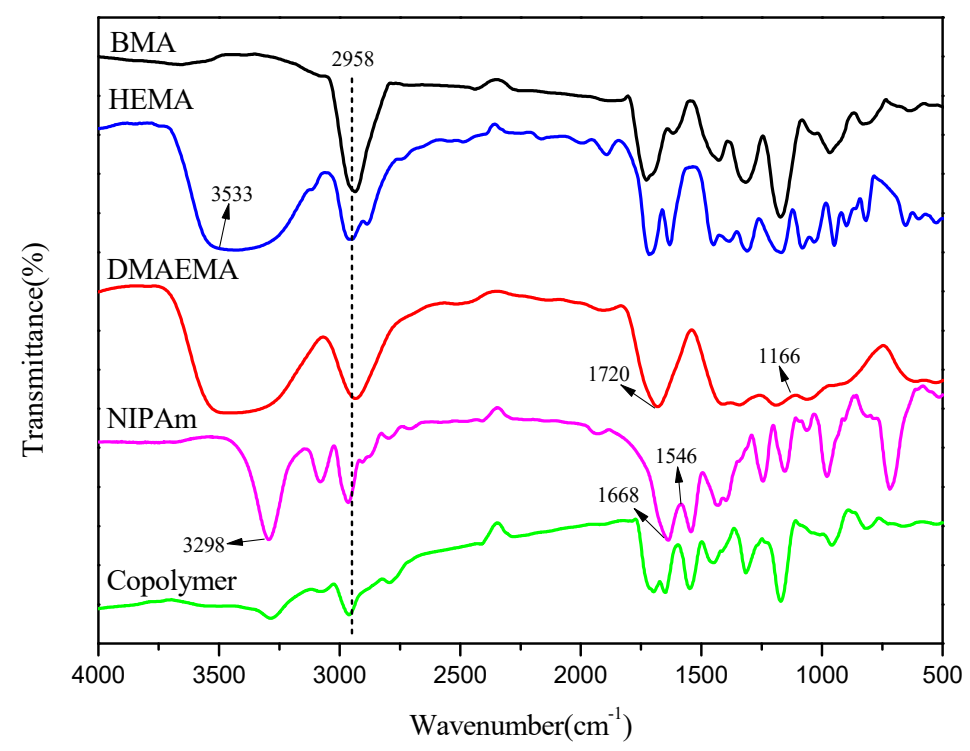

Figure 8. FTIR spectra of BMA, HEMA, DMAEMA, NIPAm and copolymer.

\subsection{Analysis of Paper Surface Elements}

The surface elements of the original paper and the copolymer-modified paper were detected through XPS. The results were analyzed to compare the changes of the surface elements of the paper before and after modification, which proved that the copolymer attached to the paper successfully and played a role in the modification of the paper. As shown by the black curve in Figure 9a, the surface of the original paper was mainly composed of a C element centered on $282.6 \mathrm{eV}$ and an O element around $530.9 \mathrm{eV}$. The red curve could be found and the modified paper surface had a new $\mathrm{N}$ peak at the center of $396.8 \mathrm{eV}$. In fact, this was attributed to the amide group in the NIPAm monomer and the tertiary amine in the DMAEMA monomer. The results of the XPS testing indicated that the copolymer had successfully attached to the surface of the paper.

In addition, Figure $9 \mathrm{a}$ also showed the changes in the surface elements of the modified paper after acid (green) and alkali (blue) treatment, which could provide a basis for the $\mathrm{pH}$ response mechanism. When the modified paper was immersed in an acid solution, a $\mathrm{Cl} 2 p$ peak appeared at $194.8 \mathrm{eV}$. The $\mathrm{N} 1 \mathrm{~s}$ peaks at 396.8 and $399.5 \mathrm{eV}$ (Figure 9c) corresponded to the tertiary and quaternary amines, indicating 
that the PDMAEMA chain was protonated. When the modified paper was treated with alkali, an Na $1 \mathrm{~s}$ peak appeared at $1069.4 \mathrm{eV}$. The $\mathrm{N} 1$ s peak was only observed at $396.7 \mathrm{eV}$ (Figure 9d), which proved that PDMAEMA was deprotonated [42]. From the point of view of atomic content, the $\mathrm{N}$ atomic content of modified paper without the acid or alkali treatment was $3.3 \%$. After acid treatment, the $\mathrm{N}$ atom content of the modified paper increased to $4.3 \%$. This was caused by the quaternary amines produced by the protonation of PDMAEMA. After alkali treatment, there was no significant change in the $\mathrm{N}$ atomic content of the modified paper. This was because PDMAEMA was deprotonated and corresponded to the tertiary amine in its chain segment. This result showed that the wettability of the paper could be altered by the protonation and deprotonation processes of the PDMAEMA segment in different $\mathrm{pH}$ solutions.
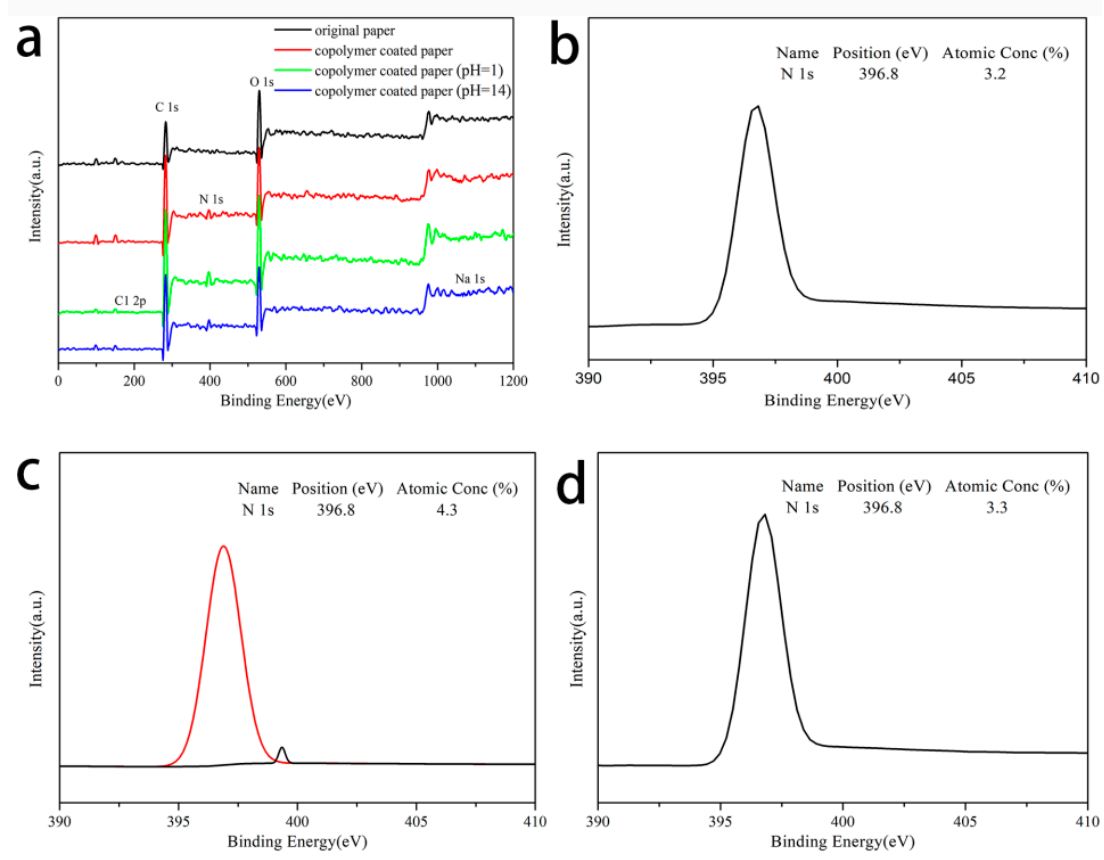

Figure 9. (a) XPS spectra. N 1s core level of (b) copolymer coated paper, (c) modified paper treated with acid, and (d) modified paper treated with an alkali, respectively.

\subsection{Thermal Analysis of Copolymer and Paper}

\subsubsection{Thermal Analysis of Copolymer}

The DSC curve of the temperature and $\mathrm{pH}$ dual-responsive copolymer was shown in Figure 10. The phase transition temperature, LCST, was taken as the peak point at which the heat flow changes during the heating process [43]. It can be found from the figure that the phase transition temperature of the copolymer is approximately $26.5^{\circ} \mathrm{C}$. As mentioned previously, the LCST of PNIPAm is around $32-34^{\circ} \mathrm{C}$. With the change in temperature, PNIPAm chains undergo reversible contraction and expansion. Above LCST, PNIPAm exhibits hydrophobicity. Below LCST, PNIPAm exhibits hydrophilicity. However, this value varies when PNIPAm is used in the copolymer form rather than a homopolymer. Because the nature of the monomer copolymerized with NIPAm will affect the phase transition temperature and temperature sensitivity. In this study, BMA and HEMA were used as comonomers to participate in the polymerization reaction. The decrease of LCST might be attributed to the presence of poly (HEMA) and poly (BMA) in the prepared copolymer. It could be explained by the weakening of the hydrogen bond between the copolymer and the solvent water and the strengthening of the hydrophobic bond between the polymer chains. The -OH of HEMA not only form hydrogen bonds among themselves but also get hydrogen bonded with the amide group (-CONH) of NIPAm. This consequently leads to the copolymer exhibiting more hydrophobicity than the homopolymer of 
pure PNIPAm [44]. This causes a shifting of the LCST to the lower side. Besides, studies have found that when PNIPAm was copolymerized with a hydrophobic polymer (poly (BMA)), the LCST of the resulting copolymer was reduced [45].

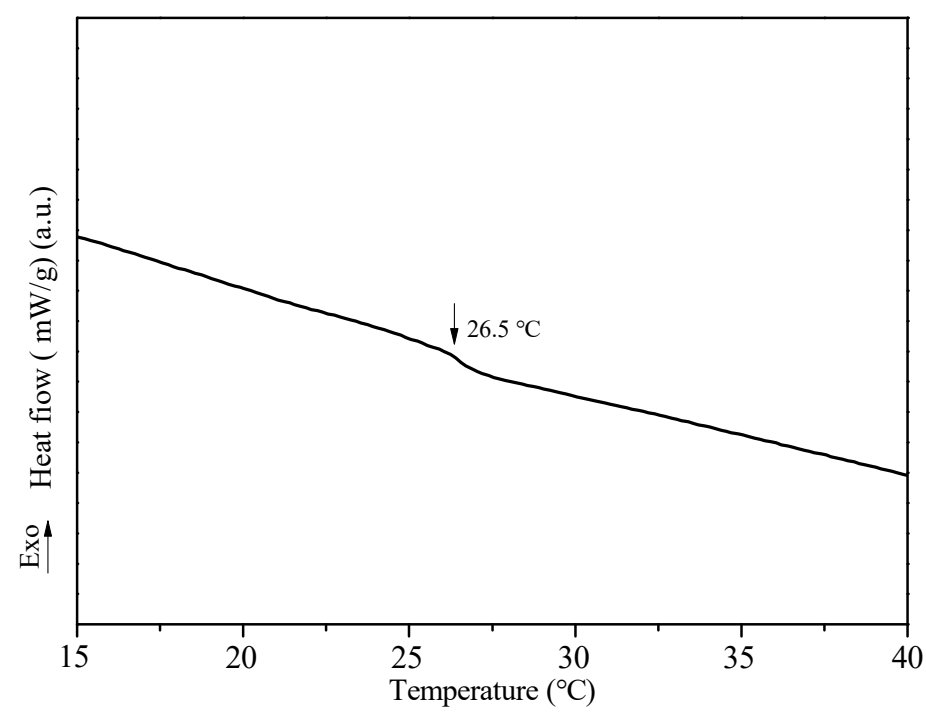

Figure 10. The DSC curve of the copolymer (PBMA-co-PHEMA-co-PDMAEMA-co-PNIPAm).

\subsubsection{Thermal Analysis of Paper}

The two curves in Figure 11 show the weight loss of the original paper (black) and the modified paper (red) as they continue to heat up from room temperature to $700{ }^{\circ} \mathrm{C}$. It could be seen from the figure that before $150{ }^{\circ} \mathrm{C}$, the weight loss of the two papers accounts for about $5 \%$ of the total mass. This was due to the weight loss caused by the desorption of the cellulose which physically adsorbed water in the paper. After $150{ }^{\circ} \mathrm{C}$, the weight loss of the original paper in the temperature range of $280-370{ }^{\circ} \mathrm{C}$ was caused by the pyrolysis of cellulose to produce small molecular gases and large molecular volatile components. The weight loss was most obvious at this stage, accounting for about $75 \%$ of the total mass. The weight loss after $370{ }^{\circ} \mathrm{C}$ was mainly due to the slow decomposition of residual materials. During the entire heating process, the weight of the unmodified paper dropped from $100 \%$ to about $20 \%$, and a total of about $80 \%$ of the weight was lost. The weight loss of the modified paper was most obvious in the temperature range of $260-355{ }^{\circ} \mathrm{C}$, accounting for $72 \%$ of the total mass. This was mainly because of the pyrolysis of cellulose to produce small molecules of gas and large molecules of volatile matter. The main pyrolysis temperature of the modified paper started at $260{ }^{\circ} \mathrm{C}$, which was $20^{\circ} \mathrm{C}$ lower than the original paper. This phenomenon could be caused by the increased surface roughness of the modified paper after the copolymer was coated. When the temperature increased, the heating area of the modified paper was larger and more uniform than that of the original paper, so the modified paper would lose weight first at a relatively low temperature. The weight loss of modified paper after $355^{\circ} \mathrm{C}$ was also because of the slow decomposition of the paper fiber residue. It was found that under the same conditions, the final weight of paper after pyrolysis was about $23 \%$, which was about $3 \%$ more material than the original paper. The extra weight of the paper was caused by the reactive copolymer coating on the paper surface. Therefore, from the thermal weightlessness test results, it also assisted in proving the successful coating of the copolymer on the paper surface, so that the paper was successfully modified. 


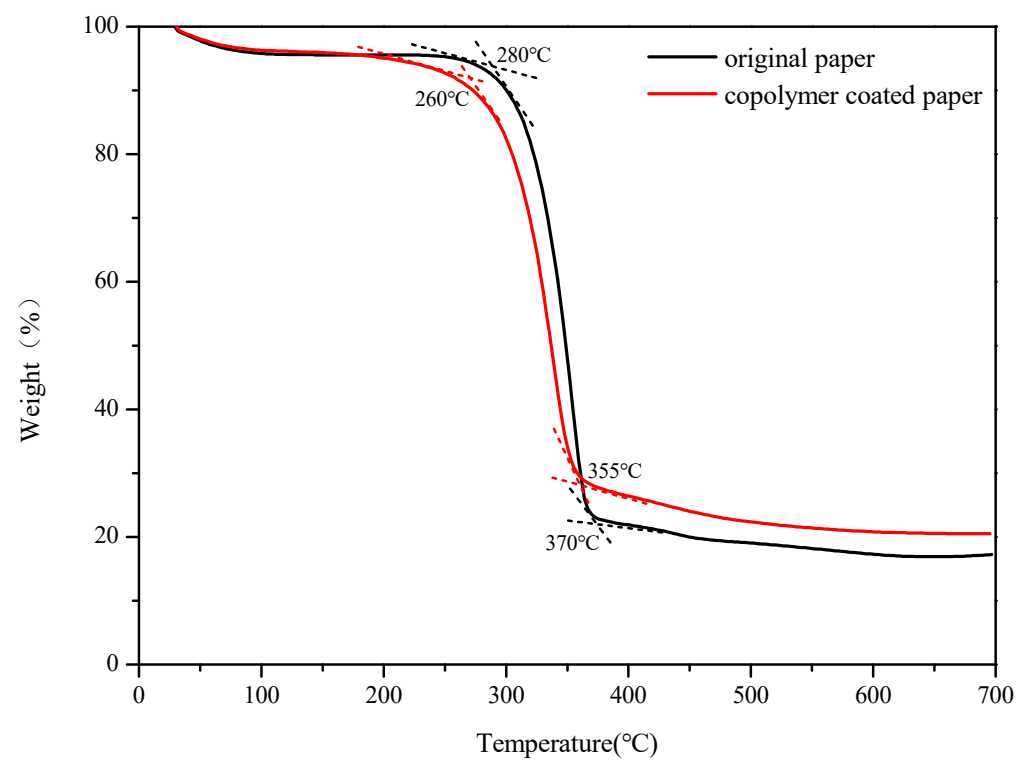

Figure 11. TGA curves of pristine paper and copolymer coated paper.

\subsection{Research on Paper Temperature Response Performance}

\subsubsection{Determination of LCST for the Dual-Responsive Superhydrophobic Paper}

According to the oil-water separation experiment between the sample bottle and the beaker device, as shown in Figure 12, when the temperature of the oil-water mixture was 10 and $20^{\circ} \mathrm{C}$ respectively, deionized water was collected in the bottle, while n-hexane stained with oil red $\mathrm{O}$ was left in the beaker. This indicated that the paper was hydrophilic and oleophobic after the two temperature treatments. When the temperature of the oil-water mixture was 35,50 and $65{ }^{\circ} \mathrm{C}$ respectively, red n-hexane was collected in the sample bottle and deionized water was left in the beaker. This indicated that the paper remained in a hydrophobic and lipophilic state at these three temperatures. Therefore, it could be determined that the LCST range of the prepared dual-responsive superhydrophobic paper was 20 to $35{ }^{\circ} \mathrm{C}$.

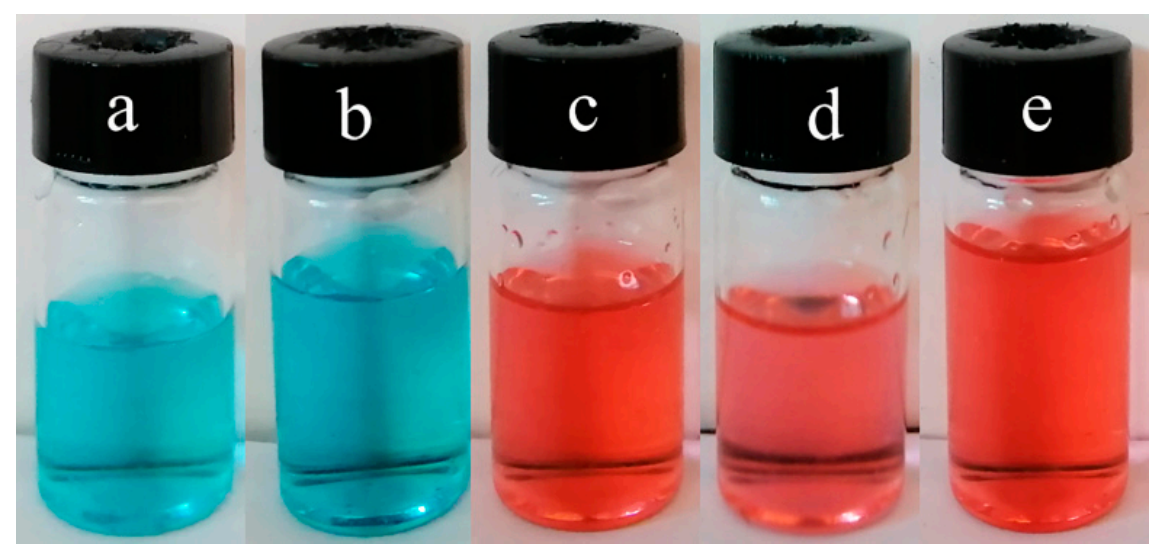

Figure 12. Liquid collected by oil and water separation at different temperatures: $(\mathbf{a}, \mathbf{b})$ water: (a) $10{ }^{\circ} \mathrm{C}$; (b) $20{ }^{\circ} \mathrm{C}$; (c-e) hexane: (c) $35^{\circ} \mathrm{C}$; (d) $50{ }^{\circ} \mathrm{C}$; (e) $65^{\circ} \mathrm{C}$.

Next, in the temperature range of 20 to $35^{\circ} \mathrm{C}$, with a temperature gradient difference of $5{ }^{\circ} \mathrm{C}$, an oil-water mixture at 25 and $30^{\circ} \mathrm{C}$ was prepared to repeat the oil-water separation experiment, and so on. According to the experimental results, the LCST of the dual-responsive superhydrophobic 
paper in this experiment was finally obtained at $25^{\circ} \mathrm{C}$. This is basically consistent with the LCST value $\left(26.5^{\circ} \mathrm{C}\right)$ of the copolymer. It further shows that the paper is modified successfully.

\subsubsection{Temperature Response Testing and Analysis}

After the LCST of the modified paper was determined, the temperature response of the paper surface under different conditions could be tested by measuring the contact angle of the paper treated at different temperatures. The specific method was as follows: two successfully modified superhydrophobic papers were treated at two temperatures below and above the LCST, respectively. One of them used an ice bath to treat the paper at $15^{\circ} \mathrm{C}$, while the other used a hot bath to treat the paper at $55^{\circ} \mathrm{C}$. As shown in Figure 13a, the contact angle of the paper surface changes with time below $55^{\circ} \mathrm{C}$. When the paper was above the LCST, the initial state of the paper was superhydrophobic, and its contact angle was $161^{\circ}$. After $3 \mathrm{~h}$, it remained superhydrophobic, and the contact angle at this time was still greater than $150^{\circ}$, which was $157^{\circ}$. As shown in Figure $13 \mathrm{~b}$, when the paper was processed at $15^{\circ} \mathrm{C}$, the surface contact angle of the paper reached $158^{\circ}$ at the beginning, which was a superhydrophobic state. After a few seconds, the water droplets on the surface of the paper began to slowly penetrate the paper, and the contact angle decreased gradually. Within $30 \mathrm{~s}$, the water droplets had completely penetrated the surface of the paper, and the water contact angle had reduced to $0^{\circ}$. At this time, the paper successfully transformed into hydrophilic paper. The results of the temperature response test show that the modified superhydrophobic paper has a theoretical temperature response performance. It is superhydrophobic paper under conditions higher than the LCST and it will transform into superhydrophilic paper when it is below the LCST.

The temperature-responsiveness of the modified paper is due to the addition of a temperature-responsive polymer PNIPAm. PNIPAm has been thoroughly studied [46-48]. According to the results, PNIPAm exhibits a superhydrophobic performance when the ambient temperature is higher than the LCST of the polymer. When the outside temperature is lower than LCST, it will become hydrophilic.

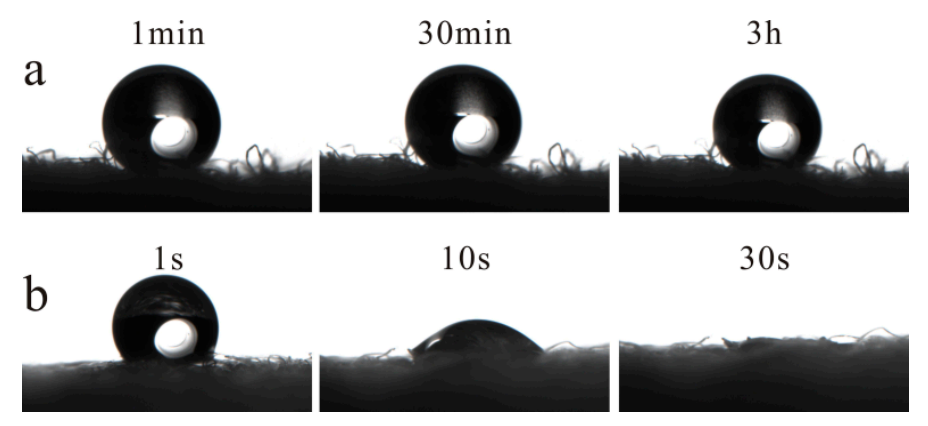

Figure 13. The contact angles of paper at different temperature: (a) $\mathrm{T}\left(55^{\circ} \mathrm{C}\right)>\mathrm{LCST}$; (b) $\mathrm{T}\left(15^{\circ} \mathrm{C}\right)$ $<$ LCST.

PNIPAm can realize the transition between superhydrophobic and hydrophilic states when the temperature changes, because the hydrogen bond between the amide group of PNIPAm and the water molecule has changed. When the temperature is lower than LCST, the $\mathrm{C}=\mathrm{O}$ and $\mathrm{N}-\mathrm{H}$ groups in PNIPAm form intermolecular hydrogen bonds with water molecules. Under the action of this hydrogen bond, the two groups above will be very closely combined with water molecules and the polymer exhibits hydrophilic properties. In addition, the effect of hydrogen bond will lead to hydration expansion of PNIPAm, which provides sufficient power for the PNIPAm chain segment on the surface to cover other polymers, thus making PNIPAm dominant and contributing to hydrophilicity on its surface. On the contrary, when the temperature reaches above the LCST, as the temperature increases, the hydrogen bonds between the molecules will gradually weaken, making the hydrophobic interaction between the isopropyl groups more obvious. When the temperature rises to a certain degree, 
the hydrogen bond between the $\mathrm{C}=\mathrm{O}$ and $\mathrm{N}-\mathrm{H}$ groups and water molecules will be broken, and an intramolecular hydrogen bond will be formed. The PNIPAm at this time shrinks compared with the state under the LCST, causing it to be dehydrated and collapsed. In this way, its hydrophobic groups and other hydrophobic polymers used in polymerization reaction (such as PBMA and PDMAEMA, etc.) are exposed, so that the surface of the prepared polymer shows superhydrophobic characteristics. The analysis of the temperature response mechanism is shown in Figure 14.

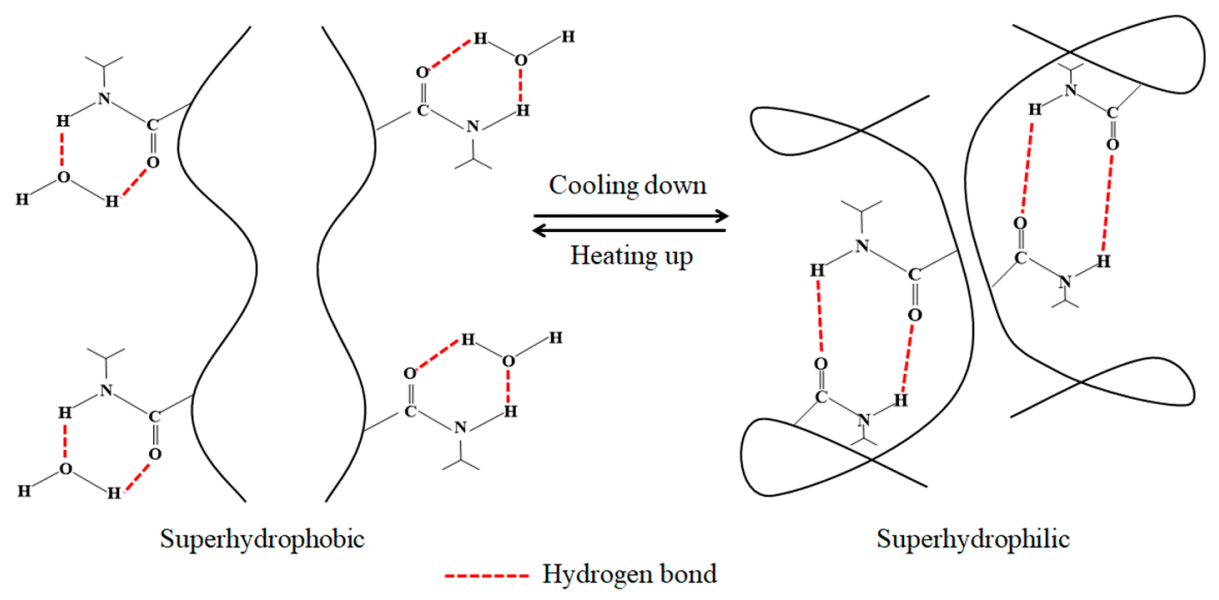

Figure 14. Mechanism of temperature response.

\subsection{Study and Analysis of pH-Response}

Similarly, the $\mathrm{pH}$-responsive performance of the prepared superhydrophobic paper was tested to verify whether it successfully possessed a $\mathrm{pH}$-response. Figure 15a shows that the initial contact angle of the paper was greater than $150^{\circ}$ and up to $159^{\circ}$ after the paper was infiltrated by an acidic solution with $\mathrm{pH} 1$. However, after a short time, the water droplets on the surface began to permeate the paper slowly. The second picture of Figure 15a shows the state of water droplets on the surface of the paper after $30 \mathrm{~s}$. As the time continued to increase, the water droplets on the surface of the paper disappeared completely after $1 \mathrm{~min}$. The contact angle was obviously dropped to $0^{\circ}$. After soaking the modified superhydrophobic paper in a neutral solution with a $\mathrm{pH}$ of 7 and an alkaline solution with a $\mathrm{pH}$ of 14 , the original superhydrophobic state had been maintained for $3 \mathrm{~h}$. The test results of $\mathrm{pH}$ responsiveness show that the superhydrophobic paper prepared in this research had good $\mathrm{pH}$ responsiveness. After acid treatment, the paper would gradually change from a superhydrophobic state to a hydrophilic state. But under neutral and alkaline conditions, its superhydrophobic state could remain unchanged and had a good stability. This shows that the superhydrophobic paper can flexibly switch between superhydrophobic and superhydrophilic states when the external $\mathrm{pH}$ value changes.

Among many external stimuli, due to the simple operation when responding to $\mathrm{pH}$ stimuli, no special facilities are needed. $\mathrm{pH}$ stimulation is more advantageous and attractive in applied research. In theory, most reactive materials that can respond to external $\mathrm{pH}$ stimuli have ionizable groups, such as sulfonates, carboxylic acids, pyridines and amines, which respond to changes in the $\mathrm{pH}$ of each repeating unit. 


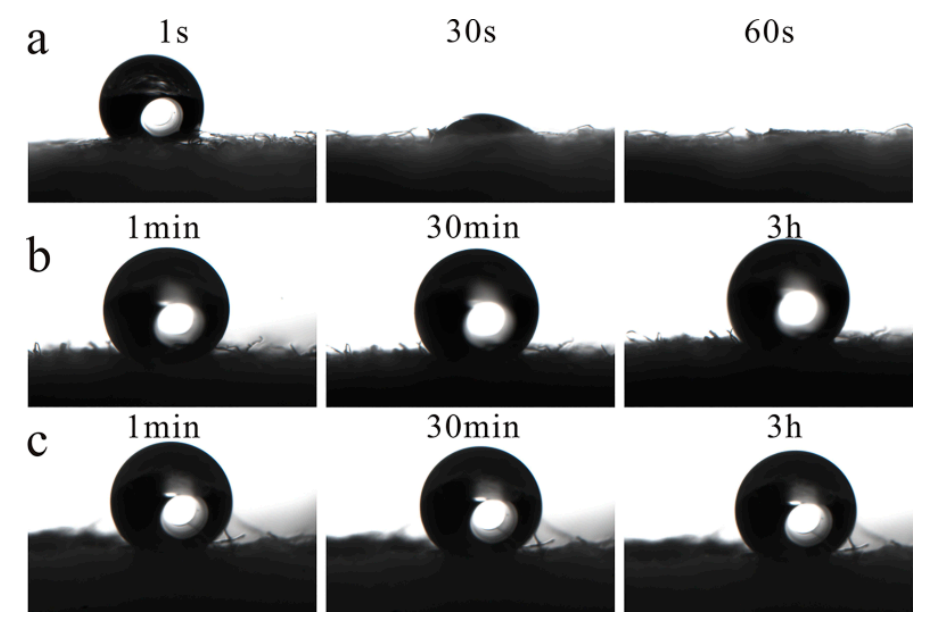

Figure 15. The contact angles of paper at different $\mathrm{pH}$ conditions: $(\mathbf{a}) \mathrm{pH}=1 ;(\mathbf{b}) \mathrm{pH}=7 ;(\mathbf{c}) \mathrm{pH}=14$.

In this study, poly (2-dimethylamino) ethyl methacrylate (PDMAEMA) was selected as the $\mathrm{pH}$-responsive polymer. It is a sensitive polymer material with $\mathrm{pH}$ response. PDMAEMA has ionizable tertiary amine groups. This allows it to undergo a protonation or deprotonation transition when the external $\mathrm{pH}$ value changes, so as to react accordingly $[49,50]$. When the prepared copolymer is under an acidic environment, the tertiary amine group in PDMAEMA will be protonated to form a positively charged ammonium group $\left(\mathrm{QA}^{+}\right)$. The molecular hydrogen bonds between the groups will combine with the surrounding water molecules to form a layer of water molecules on the surface, which increases the surface energy of the material and makes the material exhibit hydrophilic properties. At the same time, this layer of water molecules can block the entry of oil and make the material exhibit oleophobic properties. When the material is in a neutral and alkaline environment, the hydroxyl group in the solution attacks the nitrogen atom of $\mathrm{N}^{+}-\mathrm{H}$ and releases a proton at the same time. The chloride ion in the quaternary ammonium salt is removed, and the ionizing group can be deprolongated. At this time, the surface wettability of tertiary amine groups is dominated by hydrophobic groups, and hydrophobic interaction becomes the major force. This leads to the superhydrophobicity of the copolymer. In other words, the protonation and deprotonation reactions of PDMAEMA cause a significant change in the wettability of the material surface. The analysis of the $\mathrm{pH}$-response mechanism is shown in Figure 16.

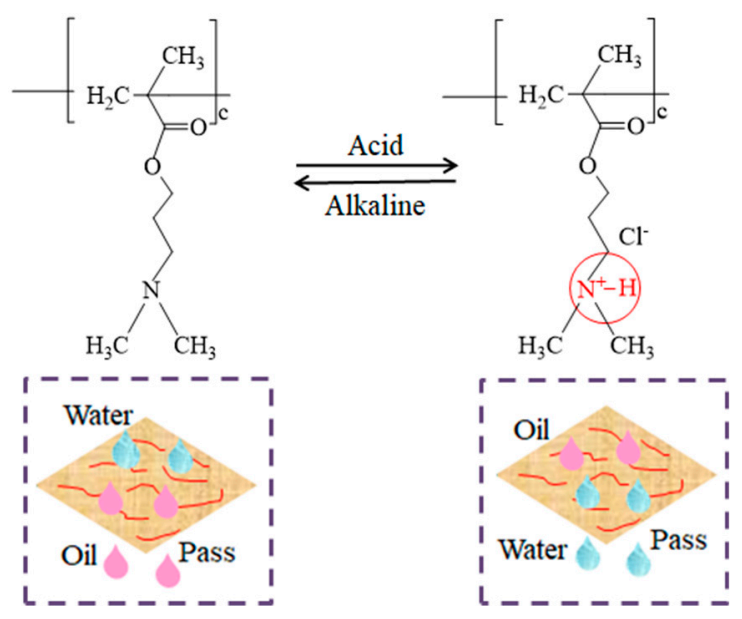

Figure 16. Mechanism of $\mathrm{pH}$-response.

\section{Conclusions}

In this study, we used the dip-coating method to immerse the pretreated paper in the copolymer solution to prepare a superhydrophobic paper with dual-responsiveness of temperature and $\mathrm{pH}$. 
Analysis such as ${ }^{1} \mathrm{H}$ NMR, FTIR and SEM found that the copolymer had successfully synthesized and was uniformly coated on the paper. Static water contact angle and oil-water separation tests showed that the functionalized paper could make corresponding hydrophilic/hydrophobic reactions to temperature and $\mathrm{pH}$ changes. When the paper was in the conditions of $\mathrm{pH} \geq 7$ and $T>25^{\circ} \mathrm{C}$, it showed good superhydrophobic performance, otherwise it would change to a hydrophilic state. The dual-responsive superhydrophobic paper prepared in this research successfully realized the conversion of the hydrophilic/hydrophobic state to different temperatures and $\mathrm{pHs}$. This not only extends the application range of superhydrophobic paper in moisture-proof packaging, paper-based sensors, oil-water separation, and self-cleaning materials, but is also a good reference for the significance of the in-depth research into the current intelligent responses to superhydrophobic paper.

Author Contributions: Data curation, B.D. and D.X.; Formal analysis, R.L. and H.L.; Funding acquisition, S.Z.; Methodology, B.D. and D.X.; Writing—original draft, B.D. and D.X.; Writing—review \& editing, K.Y. All authors have read and agreed to the published version of the manuscript.

Funding: This work was supported in part by NSF of the Science and Technology Department of Shaanxi Province under Grant Nos. 2019JM-122, Doctoral Research Initiation Fund of Xi'an University of Technology under Grant Nos. 108-451119007, NSF of the Key Laboratory of Shaanxi Provincial Department of Education under Grant Nos. 15JS075, Xi'an Science and technology plan project under Grant Nos. GXYD14.27 and Key scientific research program of Shaanxi Provincial Department of Education under Grant Nos. 20JY055.

Acknowledgments: We acknowledge the support by Shaanxi Collaborative Innovation Center of Green Intelligent Printing and Packaging.

Conflicts of Interest: The authors declare no conflict of interest.

\section{References}

1. Pan, Y.L.; Liu, L.M.; Zhang, Z.J. Surfaces with controllable super-wettability and applications for smart oil-water separation. Chem. Eng. J. 2019, 378, 1-8. [CrossRef]

2. Tian, Y.; Su, B.; Jiang, L. Interfacial Material System Exhibiting Superwettability. Adv. Mater. 2014, 26, 6872-6897. [CrossRef] [PubMed]

3. Liu, M.J.; Wang, S.T.; Jiang, L. Bioinspired multiscale surfaces with special wettability. MRS Bull. 2013, 38, 375-382. [CrossRef]

4. Roach, P.; Shirtcliffe, N.J.; Newton, M.I. Progess in superhydrophobic surface development. Soft Matter 2008, 4, 224-240. [CrossRef] [PubMed]

5. Verho, T.; Bower, C.; Andrew, P. Mechanically durable superhydrophobic surfaces. Adv. Mater. 2011, 23, 673-678. [CrossRef] [PubMed]

6. Barthwal, S.; Barthwal, S.; Singh, B.; Singh, N.B. Multifunctional and fluorine-free superhydrophobic composite coating based on PDMS modified MWCNTs/ZnO with self-cleaning, oil-water separation, and flame retardant properties. Colloids Surf. A 2020, 597, 1-25. [CrossRef]

7. Tang, L.; Wang, G.; Zeng, Z.X. Three-dimensional adsorbent with $\mathrm{pH}$ induced superhydrophobic and superhydrophilic transformation for oil recycle and adsorbent regeneration. J. Colloid Interface Sci. 2020, 575, 231-244. [CrossRef]

8. Liang, Y.Y.; Ju, J.G.; Deng, N.P. Super-hydrophobic self-cleaning bead-like $\mathrm{SiO}_{2} @ P T F E$ nanofiber membranes for waterproof-breathable applications. Appl. Surf. Sci. 2018, 442, 54-64. [CrossRef]

9. Long, W.J.; Li, H.N.; Yang, B. Superhydrophobic diamond-coated Si nanowires for application of anti-biofouling. J. Mater. Sci. Technol. 2020, 48, 1-8. [CrossRef]

10. Zhang, M.L.; Yu, J.; Chen, R.R.; Liu, Q.; Liu, J.Y.; Song, D.L.; Liu, P.L.; Gao, L.T.; Wang, J. Highly transparent and robust slippery lubricant-infused porous surfaces with anti-icing and anti-fouling performances. J. Alloy. Compd. 2019, 803, 51-60. [CrossRef]

11. Varshney, P.; Mohapatra, S.S. Durable and regenerable superhydrophobic coatings for brass surfaces with excellent self-cleaning and anti-fogging properties prepared by immersion technique. Tribol. Int. 2018, 123, 17-25. [CrossRef]

12. Yang, C.J.; Wang, M.; Yang, Z. Investigation of effects of acid, alkali, and salt solutions on fluorinated superhydrophobic surfaces. Langmuir 2019, 35, 17027-17036. [CrossRef] [PubMed] 
13. Esmaeili, A.R.; Mir, N.; Mohammadi, R. A facile, fast, and low-cost method for fabrication of micro/nano-textured superhydrophobic surfaces. J. Colloid Interface Sci. 2020, 573, 317-327. [CrossRef] [PubMed]

14. Yang, Z.; Liu, X.P.; Tian, Y.L. A contrastive investigation on anticorrosive performance of laser-induced super-hydrophobic and oil-infused slippery coatings. Prog. Org. Coat. 2019, 138, 1-10. [CrossRef]

15. Nokes, J.M.; Liedert, R.; Kim, M.Y. Reduced blood coagulation on roll-to-roll, shrink-induced superhydrophobic plastics. Adv. Healthc. Mater. 2016, 5, 593-601. [CrossRef] [PubMed]

16. Su, X.J.; Li, H.Q.; Lai, X.J. Dual-functional superhydrophobic textiles with asymmetric roll-down/pinned states for water droplet transportation and oil-water separation. ACS Appl. Mater. Interfaces 2018, 10, 4213-4221. [CrossRef] [PubMed]

17. Liu, F.; Wang, S.L.; Zhang, M. Improvement of mechanical robustness of the superhydrophobic wood surface by coating PVA/SiO ${ }_{2}$ composite polymer. Appl. Surf. Sci. 2013, 280, 686-692. [CrossRef]

18. Yang, Y.; He, H.; Li, Y.; Qiu, J. Using nanoimprint lithography to create Robust, Buoyant, superhydrophobic $\mathrm{PVB} / \mathrm{SiO}_{2}$ coatings on wood surfaces inspired by red roses petal. Sci. Rep. 2019, 9, 9961. [CrossRef]

19. Chang, B.; Zhou, Q.; Ras, R.H.A. Sliding droplets on hydrophilic/superhydrophobic patterned surfaces for liquid deposition. Appl. Phys. Lett. 2016, 108, 1-4. [CrossRef]

20. Zhang, X.; Batchelor, W.; Shen, W. Building dual-scale roughness using inorganic pigments for fabrication of super-hydrophobic paper. Ind. Eng. Chem. Res. 2017, 56, 3618-3628. [CrossRef]

21. Xu, C.L.; Song, F.; Wang, X.L.; Wang, Y.Z. Surface modification with hierarchical CuO arrays toward a flexible, durable superhydrophobic and self-cleaning material. Chem. Eng. J. 2017, 313, 1328-1334. [CrossRef]

22. Li, H.; Yang, J.; Li, P. A facile method for preparation superhydrophobic paper with enhanced physical strength and moisture-proofing property. Carbohydr. Polym. 2017, 160, 9-17. [CrossRef] [PubMed]

23. An, Q.B.; Gan, S.Y.; Xu, J.N. A multichannel electrochemical all-solid-state wearable potentiometric sensor for real-time sweat ion monitoring. Electrochem. Commun. 2019, 107, 1-7. [CrossRef]

24. Xi, G.Q.; Li, J.F.; Deng, H.; Ma, M.G. Synthesis of durable superhydrophobic coating and its applications in oil/water separation. Sci. Adv. Mater. 2020, 12, 676-684. [CrossRef]

25. Cao, Y.J.; Salvini, A.; Camaiti, M. Current status and future prospects of applying bioinspired superhydrophobic materials for conservation of stone artworks. Coatings 2020, 10, 353. [CrossRef]

26. Ruan, X.W.; Xu, T.C.; Chen, D.J. Superhydrophobic paper with mussel-inspired polydimethylsiloxane-silica nanoparticle coatings for effective oil/water separation. RSC Adv. 2020, 10, 8008-8015. [CrossRef]

27. Li, R.Q.; Zhang, G.L.; Wang, J.F. Superwetting pH-responsive polyaniline coatings: Toward versatile separation of complex oil-water mixtures. Langmuir 2020, 36, 760-768. [CrossRef]

28. Liu, P.F.; Zhang, Y.P.; Liu, S.Q. Bio-inspired fabrication of fire-retarding, magnetic-responsive, superhydrophobic sponges for oil and organics collection. Appl. Clay Sci. 2019, 172, 19-27. [CrossRef]

29. Fu, S.D.; Zhou, H.; Wang, H.X. Magnet-responsive, superhydrophobic fabrics from waterborne, fluoride-free coatings. RSC Adv. 2018, 8,717-723. [CrossRef]

30. Chen, K.L.; Zhou, S.X.; Yang, S.; Wu, L.M. Fabrication of all-water-based self-repairing superhydrophobic coatings based on UV-responsive microcapsules. Adv. Funct. Mater. 2015, 25, 1035-1041. [CrossRef]

31. Liu, Z.A.; Yang, X.L.; Pang, G.B. Temperature-based adhesion tuning and superwettability switching on superhydrophobic aluminum surface for droplet manipulations. Surf. Coat. Technol. 2019, 375, 527-533. [CrossRef]

32. de Leon, A.; Advincula, R.C. Reversible superhydrophilicity and superhydrophobicity on a lotus-leaf pattern. ACS Appl. Mater. Interfaces 2014, 6, 22666-22672. [CrossRef] [PubMed]

33. Kota, A.K.; Li, Y.X.; Mabry, J.M.; Tuteja, A. Hierarchically structured superoleophobic surfaces with ultralow contact angle hysteresis. Adv. Mater. 2012, 24, 5838-5843. [CrossRef] [PubMed]

34. Song, J.; Janczewski, D.; Ma, Y.J.; Hempenius, M.; Xu, J.W.; Vancso, G.J. Redox-controlled release of molecular payloads from multilayered organometallic polyelectrolyte films. J. Mater. Chem. B. 2013, 1, 828-834. [CrossRef]

35. Abbott, N.L.; Whitesides, G.M. Potential-dependent wetting of aqueous solutions on self-assembled monolayers formed from 15-(Ferrocenylcarbonyl) pentadecanethiol on gold. Langmuir 1994, 10, 1493-1497. [CrossRef]

36. Elbert, J.; Gallei, M.; Ruttiger, C.; Brunsen, A.; Didzoleit, H.; Stuhn, B.; Rehahn, M. Ferrocene Polymers for switchable surface wettability. Organometallics 2013, 32, 5873-5878. [CrossRef] 
37. Ruettiger, C.; Mehlhase, S.; Vowinkel, S.; Cherkashinin, G.; Liu, N.; Dietz, C.; Stark, R.W.; Biesalski, M.; Gallei, M. Redox-mediated flux control in functional paper. Polymer 2016, 98, 429-436. [CrossRef]

38. Chen, K.L.; Zhou, J.L.; Ge, F.Q. Smart UV-curable fabric coatings with self-healing ability for durable self-cleaning and intelligent oil/water separation. Colloids Surf. A 2019, 565, 86-96. [CrossRef]

39. Cheng, Z.J.; Lai, H.; Du, M. Super-hydrophobic surface with switchable adhesion responsive to both temperature and $\mathrm{pH}$. Soft Matter 2012, 8, 9635-9641. [CrossRef]

40. Asatekin, A.; Mayes, A.M. Oil industry wastewater treatment with fouling resistant membranes containing amphiphilic comb copolymers. Environ. Sci. Technol. 2009, 43, 4487-4492. [CrossRef]

41. Yamada, K.; Takada, A.; Konishi, A.; Kimura, Y.; Asamoto, H.; Minamisawa, H. Hexavalent Cr ion adsorption and desorption behavior of expanded poly (tetrafluoro) ethylene films grafted with 2-(dimethylamino) ethyl methacrylate. Environ. Technol. 2019, 1-37. [CrossRef] [PubMed]

42. Dang, Z.; Liu, L.B.; Li, Y.; Xiang, Y.; Guo, G.L. In situ and ex situ pH-responsive coatings with switchable wettability for controllable oil/water separation. ACS Appl. Mater. Interfaces 2016, 8, 31281-31288. [CrossRef] [PubMed]

43. Park, J.H.; Jang, J.W.; Sim, J.H.; Kim, I.J.; Lee, D.J.; Lee, Y.H.; Park, S.H.; Kim, H.D. Preparation and properties of thermoresponsive $\mathrm{P}(\mathrm{N}$-Isopropylacrylamide-co-butylacrylate) hydrogel materials for smart windows. Int. J. Polym. Sci. 2019, 2019, 3824207. [CrossRef]

44. Jain, A.; Bajpai, J.; Bajpai, A.K.; Mishra, A. Thermoresponsive cryogels of poly (2-hydroxyethyl methacrylate-co-N-isopropyl acrylamide) (P(HEMA-co-NIPAM)): Fabrication, characterization and water sorption study. Polym. Bull. 2020, 77, 4417-4443. [CrossRef]

45. Hufendiek, A.; Trouillet, V.; Meier, M.A.R.; Barner-Kowollik, C. Temperature responsive cellulose-graft-copolymers via cellulose functionalization in an ionic liquid and RAFT polymerization. Biomacromolecules 2014, 15, 2563-2572. [CrossRef]

46. Liu, Y.W.; Sakurai, K. Thickness changes in temperature-responsive Poly (N-isopropylacrylamide) ultrathin films under ambient conditions. ACS Omega 2019, 4, 12194-12203. [CrossRef]

47. Razavi, B.; Abdollahi, A.; Roghani-Mamaqani, H.; Salami-Kalajahi, M. Light- and temperature-responsive micellar carriers prepared by spiropyran-initiated atom transfer polymerization: Investigation of photochromism kinetics, responsivities, and controlled release ofdoxorubicin. Polymer 2020, 187, 122046. [CrossRef]

48. Wang, J.; Chen, S.; Cheng, X.Y.; Peng, Q.; Li, W.K. Temperature/pH dual-responsive poly ( $N$-isopropylacrylamide)/chitosancoated luminescent composite nanospheres: Fabrication and controllable luminescence. Opt. Mater. 2018, 86, 56-61. [CrossRef]

49. Xiong, D.; Zhang, X.F.; Peng, S.Y.; Gu, H.W.; Zhang, L.J. Smart pH-sensitive micelles based on redox degradable polymers as DOX/GNPs carriers for controlled drug release and CT imaging. Colloids Surf. B 2018, 163, 29-40. [CrossRef]

50. Zhao, L.Y.; Li, L.; Wang, Y.X.; Wu, J.N.; Meng, G.H.; Liu, Z.Y.; Guo, X.H. Preparation and characterization of thermo- and $\mathrm{pH}$ dual-responsive 3D cellulose-based aerogel for oil/water separation. Appl. Phys. A 2018, 124, 9. [CrossRef]

Publisher's Note: MDPI stays neutral with regard to jurisdictional claims in published maps and institutional affiliations.

(C) 2020 by the authors. Licensee MDPI, Basel, Switzerland. This article is an open access article distributed under the terms and conditions of the Creative Commons Attribution (CC BY) license (http://creativecommons.org/licenses/by/4.0/). 